
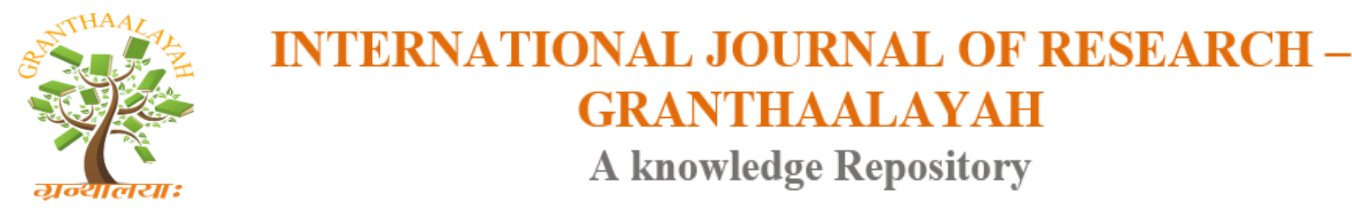

Science

\title{
ON LACUNARY ARITHMETIC STATISTICAL CONTINUITY FOR DOUBLE SEQUENCES
}

\author{
M. M. Karagama ${ }^{* 1}$, F. B. Ladan ${ }^{2}$ \\ ${ }^{* 1,2}$ Department of Mathematical Sciences, University of Maiduguri, Borno State, Nigeria
}

\begin{abstract}
In this article, we shall introduce the concept of lacunary arithmetic statistical continuity for double sequences and investigate some inclusion relations.
\end{abstract}

Keywords: Summability; Arithmetic Statistical Convergence; Lacunary Arithmetic Statistical Convergence; Lacunary Arithmetic Statistical Continuity; Double Sequences.

Cite This Article: M. M. Karagama, and F. B. Ladan. (2017). "ON LACUNARY ARITHMETIC STATISTICAL CONTINUITY FOR DOUBLE SEQUENCES." International Journal of Research - Granthaalayah, 5(11), 22-26. 10.29121/granthaalayah.v5.i11.2017.2321.

\section{Introduction}

The concept of statistical convergence was introduced by Fast [4] and it was further investigated from the sequence space point of view and linked with summability theory by Fridy [2], Connor [3], Fridy and Orhan [1], Šalát [5] and many others.

While the idea of arithmetic convergence was introduced by Ruckle [9]. Yaying and Hazarika [8] used this concept of arithmetic convergence and introduced arithmetic statistical convergence and lacunary arithmetic statistical convergence of single sequence. Also Yaying and Hazarika [8] establish some sequential properties of lacunary arithmetic statistical continuity of single sequence. The concept of statistical convergence of double sequences was introduced by Mursaleen [6]. Using the method of Mursaleen, we shall extend the results of Yaying and Hazarika [8] to double sequences as follows:

\section{Lacunary Arithmetic Statistical Continuity (First we Noted)}

Definition 2.1: (Yaying and Hazarika [2017]) A sequence $x=\left(x_{k}\right)$ is called arithmetically convergent if for each $\varepsilon>0$ there is an integer $l$ such that for every integer $\mathrm{k}$ we have $\mid x_{k}-$ $x_{\langle k, l\rangle} \mid<\varepsilon$, where the symbol $\langle k, l\rangle$ denotes the greatest common divisor of two integers $k$ and $l$. We denote the sequence space of all arithmetic convergent sequence by AC. 
Definition 2.2: (Fridy and Orhan [1993]) Let $\theta=\left(k_{r}\right)$ be a lacunary sequence. A number sequence $x=\left(x_{k}\right)$ is said to be lacunary statistically convergent to $l$ or $S_{\theta}$-convergent to $l$, if, for each $\varepsilon>0$,

$\lim _{r \rightarrow \infty} \frac{1}{h_{r}}\left|\left\{k \in I_{r}:\left|x_{k}-l\right| \geq \varepsilon\right\}\right|=0$

In this case, one writes $S_{\theta}-\lim x_{k}=l$ or $x_{k} \rightarrow\left(S_{\theta}\right)$. The set of all lacunary statistically convergence sequences is denoted by $S_{\theta}$

Definition 2.3: (Yaying and Hazarika [2017]) A sequence $x=\left(x_{k}\right)$ is said to be arithmetic statistically convergent if for each $\varepsilon>0$, there is an integer $l$ such that

$$
\lim _{n \rightarrow \infty} \frac{1}{n}\left|\left\{k \in n:\left|x_{k}-x_{\langle k, l\rangle}\right| \geq \varepsilon\right\}\right|=0
$$

We shall use ASC to denote the set of all arithmetic statistical convergent sequences. Thus for $\varepsilon>0$ and integer 1

$$
\operatorname{ASC}=\left\{\left(x_{k}\right): \lim _{n \rightarrow \infty} \frac{1}{n}\left|\left\{k \in n:\left|x_{k}-x_{\langle k, l\rangle}\right| \geq \varepsilon\right\}\right|=0\right\}
$$

We shall write $A S C-\lim x_{k}=x_{\langle k, l\rangle}$ to denote the sequence $\left(x_{k}\right)$ is arithmetic statistically convergent to $x_{\langle k, l\rangle}$.

Definition 2.4: (Yaying and Hazarika [2017]) Let $\theta=\left(k_{r}\right)$ be a lacunary sequence. The number sequence $x=\left(x_{k}\right)$ is said to be lacunary arithmetic statistically convergent if for each $\varepsilon>0$ there is an integer $l$ such that

We shall write

$$
\lim _{r \rightarrow \infty} \frac{1}{h_{r}}\left|\left\{k \in I_{r}:\left|x_{k}-x_{\langle k, l\rangle}\right| \geq \varepsilon\right\}\right|=0
$$

$$
A S C_{\theta}=\left\{x=\left(x_{k}\right): \lim _{r \rightarrow \infty} \frac{1}{h_{r}}\left|\left\{k \in I_{r}:\left|x_{k}-x_{\langle k, l\rangle}\right| \geq \varepsilon\right\}\right|=0\right\} .
$$

We shall write $A S C_{\theta}-\lim x_{k}=x_{\langle k, l\rangle}$ to denote the sequence $\left(x_{k}\right)$ is lacunary arithmetic statistically convergent to $x_{\langle k, l\rangle}$.

Definition 2.5: (Yaying and Hazarika [2017]) A function $f$ defined on a subset $E$ of $\mathbb{R}$ is said to be lacunary arithmetic statistical continuous if it preserves lacunary arithmetic statistical convergence i.e. if

$$
A S C_{\theta}-\lim x_{k}=x_{\langle k, l\rangle} \text { Implies } A S C_{\theta}-\lim f\left(x_{k}\right)=f\left(x_{\langle k, l\rangle}\right)
$$

Theorem 2.1: (Yaying and Hazarika [2017]) Let $\left(f_{m}\right), m \in \mathbb{N}$ be sequence of $A S C_{\theta}$ continuous functions defined on a subset of $\mathrm{E}$ of $\mathbb{R}$ and $f_{m}$, be uniformly convergent to a function $\mathrm{f}$, then $\mathrm{f}$ is $A S C_{\theta}$ continuous. 
Theorem 2.2: (Yaying and Hazarika [2017]) The set of all $A S C_{\theta}$ continuous functions defined are on a subset $\mathrm{E}$ of $\mathbb{R}$ is a closed subset of all continuous function on $\mathrm{E}$, i.e. $\overline{A S C_{\theta}(E)}=$ $A S C_{\theta}(E)$, where $A S C_{\theta}(E)$ denotes the set of all $A S C_{\theta}$ continuous functions defined on $\mathrm{E}$ and $\overline{A S C_{\theta}(E)}$ denotes the closure of $A S C_{\theta}(E)$.

We shall now use the concept of statistical convergence to extend above concept and result to double sequences, using Analogy;

\section{Lacunary Arithmetic Statistical Continuity For Double Sequences}

Definition 3.1: A function $f$ defined on a subset $D$ of $\mathbb{R}$ is said to be lacunary arithmetic statistical continuous for double sequences if it preserves lacunary arithmetic statistical convergence for double sequences i.e. if

$$
A S C_{\theta_{r, s}}-\lim x_{k, m}=x_{\langle\langle k, l\rangle,\langle m, n\rangle\rangle} \operatorname{Implies} A S C_{\theta_{r, s}}-\lim f\left(x_{k, m}\right)=f\left(x_{\langle\langle k, l\rangle,\langle m, n\rangle\rangle}\right) .
$$

Where the symbol $\langle k, l, m, n\rangle$ denotes the greatest common divisor of four integers $k, l, m$ and $n$. We shall write $A S C_{\theta_{r, s}}$ continuous function to denote lacunary arithmetic statistical continuous for double sequences. It is easy to see that the sum and the difference of two $A S C_{\theta_{r, s}}$ continuous functions is $A S C_{\theta_{r, s}}$ continuous. Also the composition of two $A S C_{\theta_{r, s}}$ continuous functions is again $A S C_{\theta_{r, s}}$ continuous. In the classical case, it is known that the uniform limit of sequentially continuous function is sequentially continuous, now we see that the uniform limit of $A S C_{\theta_{r, s}}$ continuous functions is also $A S C_{\theta_{r, s}}$ continuous.

Theorem 3.1: Let $\left(f_{k, m}\right), k, m \in \mathbb{N}$ be sequence of $A S C_{\theta_{r, s}}$ continuous functions defined on a subset of $\mathrm{D}$ of $\mathbb{R}$ and $f_{k, m}$, be uniformly convergent to a function $\mathrm{f}$, then $\mathrm{f}$ is $A S C_{\theta_{r, s}}$ continuous.

Proof 3.1: Let $\varepsilon>0$ and $\left(x_{k, m}\right)$ be any $A S C_{\theta_{r, s}}$ convergent sequence on a subset D of $\mathbb{R}$. By the uniform convergence of $f_{k, m}$, there exist $N \in \mathbb{N}$ such that $\left|f_{k, m}(x)-f(x)\right| \leq \frac{\varepsilon}{3}$ for all $k, m \in N$ and for all $x \in D$. Since $f_{N}$ is continuous on $\mathrm{D}$, we have for an integer $l, n$.

$$
\lim _{r, s \rightarrow \infty} \frac{1}{h_{r, s}}\left|\left\{k, m \in I_{r, s}:\left|f_{N}\left(x_{k, m}\right)-f_{N}\left(x_{\langle\langle k, l\rangle,\langle m, n\rangle\rangle}\right)\right| \geq \frac{\varepsilon}{3}\right\}\right|=0
$$

On the other hand, for an integer $l, n$ we have

$$
\begin{aligned}
& \left\{k, m \in I_{r, s}:\left|f\left(x_{k, m}\right)-f\left(x_{\langle\langle k, l\rangle,\langle m, n\rangle\rangle}\right)\right| \geq \frac{\varepsilon}{3}\right\} \subset\left\{k, m \in I_{r, s}:\left|f_{N}\left(x_{k, m}\right)-f\left(x_{\langle\langle k, l\rangle,\langle m, n\rangle\rangle}\right)\right| \geq \frac{\varepsilon}{3}\right\} \\
& \cup\left\{k, m \in I_{r, s}:\left|f_{N}\left(x_{\langle\langle k, l\rangle,\langle m, n\rangle\rangle}\right)-f_{N}\left(x_{k, m}\right)\right| \geq \frac{\varepsilon}{3}\right\} \cup\left\{k, m \in I_{r, s}:\left|f_{N}\left(x_{k, m}\right)-f\left(x_{k, m}\right)\right| \geq \frac{\varepsilon}{3}\right\}
\end{aligned}
$$


Thus it follows from the above inclusion that

$$
\begin{gathered}
\lim _{r, s \rightarrow \infty} \frac{1}{h_{r, s}}\left|\left\{k, m \in I_{r, s}:\left|f\left(x_{k, m}\right)-f\left(x_{\langle\langle k, l\rangle,\langle m, n\rangle\rangle}\right)\right| \geq \varepsilon\right\}\right| \leq \\
\lim _{r, s \rightarrow \infty} \frac{1}{h_{r, s}}\left|\left\{k, m \in I_{r, s}:\left|f_{N}\left(x_{\langle\langle k, l\rangle,\langle m, n\rangle)}\right)-f\left(x_{\langle\langle k, l\rangle,\langle m, n\rangle\rangle)}\right)\right| \geq \frac{\varepsilon}{3}\right\}\right|+\lim _{r, s \rightarrow \infty} \frac{1}{h_{r, s}} \mid\{k, m \in \\
\left.I_{r, s}:\left|f_{N}\left(x_{\langle\langle k, l\rangle,\langle m, n\rangle\rangle}\right)-f_{N}\left(x_{k, m}\right)\right| \geq \frac{\varepsilon}{3}\right\}\left|+\lim _{r, s \rightarrow \infty} \frac{1}{h_{r, s}}\right|\left\{k, m \in I_{r, s}:\left|f_{N}\left(x_{k, m}\right)-f\left(x_{k, m}\right)\right| \geq \frac{\varepsilon}{3}\right\} \mid
\end{gathered}
$$

Thus, $f$ is $A S C_{\theta_{r, s}}$ continuous.

Theorem 3.2: The set of all $A S C_{\theta_{r, S}}$ continuous functions defined on a subset $\mathrm{D}$ of $\mathbb{R}$ is a closed subset of all continuous function on D, i.e. $\overline{A S C_{\theta_{r, s}}(D)}=A S C_{\theta_{r, s}}(D)$, where $\operatorname{ASC}_{\theta_{r, s}}(D)$ denotes the set of all $A S C_{\theta_{r, s}}$ continuous functions defined on D and $\overline{A S C_{\theta_{r, s}}(D)}$ denotes the closure of $A S C_{\theta_{r, s}}(D)$.

Proof 3.2: Let $\mathrm{f}$ be any element of $\overline{A S C_{\theta_{r, s}}(D)}$. Then there exist a sequence of points in $A S C_{\theta_{r, s}}(D)$ such thatlim $f_{k, m}=f$. Now let $\left(x_{k, m}\right)$ be any $A S C_{\theta_{r, s}}$ convergent sequence in D. Since $\left(f_{k, m}\right)$ converges to $f$, there exist a positive integer $N$ such that

$$
\left|f(x)-f_{k, m}(x)\right| \leq \frac{\varepsilon}{3}, \forall k, m \geq N \text { and } \forall x \in D
$$

Now $f_{N}$ is $A S C_{\theta_{r, S}}$ continuous on $\mathrm{D}$, so we have for an integer $l, n$

$$
\lim _{r, s \rightarrow \infty} \frac{1}{h_{r, s}}\left|\left\{k, m \in I_{r, s}:\left|f_{N}\left(x_{k, m}\right)-f_{N}\left(x_{\langle\langle k, l\rangle,\langle m, n\rangle)}\right)\right| \geq \frac{\varepsilon}{3}\right\}\right|=0
$$

On the other hand, for an integer $l, n$ we have

$$
\begin{gathered}
\left\{k, m \in I_{r, s}:\left|f\left(x_{k, m}\right)-f\left(x_{\langle\langle k, l\rangle,\langle m, n\rangle)}\right)\right| \geq \frac{\varepsilon}{3}\right\} \\
\subset\left|\left\{k, m \in I_{r, s}:\left|f_{N}\left(x_{\langle\langle k, l\rangle,\langle m, n\rangle\rangle}\right)-f\left(x_{\langle\langle k, l\rangle,\langle m, n\rangle}\right)\right| \geq \frac{\varepsilon}{3}\right\}\right| \\
\cup\left\{k, m \in I_{r, s}:\left|f_{N}\left(x_{\langle\langle k, l\rangle,\langle m, n\rangle\rangle}\right)-f_{N}\left(x_{k, m}\right)\right| \geq \frac{\varepsilon}{3}\right\} \\
\cup\left\{k, m \in I_{r, s}:\left|f_{N}\left(x_{k, m}\right)-f\left(x_{k, m}\right)\right| \geq \frac{\varepsilon}{3}\right\}
\end{gathered}
$$

From the above inclusion we can write

$$
\begin{gathered}
\lim _{r, s \rightarrow \infty} \frac{1}{h_{r, s}}\left|\left\{k, m \in I_{r, s}:\left|f\left(x_{k, m}\right)-f\left(x_{\langle\langle k, l\rangle,\langle m, n\rangle\rangle}\right)\right| \geq \varepsilon\right\}\right| \\
\leq \lim _{r, s \rightarrow \infty} \frac{1}{h_{r, s}}\left|\left\{k, m \in I_{r, s}:\left|f_{N}\left(x_{\langle\langle k, l\rangle,\langle m, n\rangle\rangle}\right)-f\left(x_{\langle\langle k, l\rangle,\langle m, n\rangle\rangle)}\right)\right| \geq \frac{\varepsilon}{3}\right\}\right|
\end{gathered}
$$




$$
\begin{gathered}
+\lim _{r, s \rightarrow \infty} \frac{1}{h_{r, s}}\left|\left\{k, m \in I_{r, s}:\left|f_{N}\left(x_{\langle\langle k, l\rangle,\langle m, n\rangle\rangle}\right)-f_{N}\left(x_{k, m}\right)\right| \geq \frac{\varepsilon}{3}\right\}\right| \\
+\lim _{r, s \rightarrow \infty} \frac{1}{h_{r, s}}\left|\left\{k, m \in I_{r, s}:\left|f_{N}\left(x_{k, m}\right)-f\left(x_{k, m}\right)\right| \geq \frac{\varepsilon}{3}\right\}\right|=0
\end{gathered}
$$

Thus f is $A S C_{\theta_{r, s}}$ continuous, so $f \in A S C_{\theta_{r, s}}(D)$ which gives us our required result.

\section{References}

[1] John Albert Fridy. (1993). Cihan Orhan, "LACUNARY STATISTICAL SUMMABILITY.” J. Math. Anal. Appl. 173(2) 497-504.

[2] John Albert Fridy. (1985). “ON STATISTICAL CONVERGENCE.” Analysis, 5, 301-313.

[3] J.S. Connor. (1988). "THE STATISTICAL AND STRONG P-CESARO CONVERGENCE OF SEQUENCES.” Analysis, 8, 47-63., ISSN (Online) 2196-6753, ISSN (Print) 0174-4747, DOI: https://doi.org/10.1524/anly.1988.8.12.47.

[4] H. Fast. (1951). "SUR LA CONVERGENCE STATISTIQUE." Colloq Math, 2, 241-244.

[5] T. Šalát. (1980). “ON STATISTICALLY CONVERGENT SEQUENCES OF REAL NUMBERS.” Math Slovaca, 30, 139-15

[6] Mohammad Mursaleen, and O. H. H. Edely. (2003). "STATISTICAL CONVERGENCE OF DOUBLE SEQUENCES.” J. Math.Anal.Appl., 288, 223-231.

[7] A. R. Freedman, J. J. Sember, M. Raphael. (1978). "SOME CESÁRO-TYPE SUMMABILITY SPACES." Proc. Lond. Math. Soc. 37 508-520.

[8] Taja Yaying and Bipan Hazarika. (2017). "LACUNARY ARITHMETIC STATISTICAL CONVERGENCE.” arXiv:1703.03780v1 [Math.GM], 8 Mar.

[9] W. H. Ruckle. (2012). “ARITHMETICAL SUMMABILITY.” Jour. Math. Anal. Appl. 396 741748.

[10] John Albert Fridy, and Cihan Orhan. (1993). "LACUNARY STATISTICAL CONVERGENCE." Pacific Journal of Mathematics, 160, no. 1, 43-53.

*Corresponding author.

E-mail address: mustaphakaragama @gmail.com 